\title{
Estandarización de un método basado en espectrofotometría para evaluar vitamina A en suero y en aguaje
}

\author{
José Olivera, Ivonne Reyes, Rubén Valdivieso, Rosa Oriondo, Manuel Taboada
}

Centro de Investigación de Bioquímica y Nutrición, Facultad de Medicina, UNMSM

Objetivos: Medir vitamina A en suero y en pulpa de aguaje, según las condiciones de nuestro laboratorio.

Diseño: Exploratorio.

Institución: Centro de Investigación de Bioquímica y Nutrición, Facultad de Medicina, UNMSM.

Material biológico: Pulpa de aguaje del mercado local de Rioja y suero de los participantes del estudio.

Intervenciones: Se realizó tres repeticiones de ensayos mínimos. Según la metodología seleccionada (luego de una búsqueda bibliográfica), se usó los valores de sus resultados para ser comparados con los obtenidos en nuestros ensayos. La determinación de vitamina A a nivel sérico se realizó con la técnica basada en la destrucción de la vitamina A por luz UV. En la pulpa de aguaje, se aplicó la metodología que mide equivalente de retinol a partir de un extracto carotinoide, determinándose longitud de máxima absorción a $436 \mathrm{~nm}$ y el valor de extinción molar.

Principales medidas de resultados: Medición de vitamina A en suero y en pulpa de aguaje.

Resultados: Para el suero, no se consiguió resultados concluyentes. En pulpa de aguaje se obtuvo el valor de 1,23 uM de retinol (equivalente a $7.435 \mathrm{uM} / 100 \mathrm{~g}$ ), comparándolo con datos de tablas de alimentos.

Conclusiones: Se consiguió medir vitamina A en extracto carotenoide de pulpa de aguaje en equivalente de retinol, aplicación útil para estudios preliminares de vitamina A en fruta antes de aplicar métodos sofisticados.

Palabras clave: Vitamina A, espectrofotometría, pulpa de Mauritia flexuosa. 